Bull. Austral. Math. Soc.

VOL. 44 (1991) [429-450]

\title{
SMALL SQUARING AND CUBING PROPERTIES FOR FINITE GROUPS
}

\author{
Ya. G. Berkovich, G.A. Freiman and Cheryl E. Praeger
}

\begin{abstract}
A group $G$ is said to have the small squaring property on $k$-sets if $\left|K^{2}\right|<k^{2}$ for all $k$-element subsets $K$ of $G$, and is said to have the small cubing property on $k$-sets if $\left|K^{3}\right|<k^{3}$ for all $k$-element subsets $K$. It is shown that a finite nonabelian group with the small squaring property on 3-sets is either a 2-group or is of the form $T P$ with $T$ a normal abelian odd order subgroup and $P$ a nontrivial 2-group such that $Q=C_{P}(T)$ has index 2 in $P$ and $P$ inverts $T$. Moreover either $P$ is abelian and $Q$ is elementary abelian, or $Q$ is abelian and each element of $P-Q$ inverts $Q$. Conversely each group of the form $T P$ as above has the small squaring property on 3-sets. As for the nonabelian 2-groups with the small squaring property on 3-sets, those of exponent greater then 4 are classified and the examples are similar to dihedral or generalised quaternion groups. The remaining classification problem of exponent 4 nonabelian examples is not complete, but these examples are shown to have derived length 2 , centre of exponent at most 4 , and derived quotient of exponent at most 4. Further it is shown that a nonabelian group $G$ satisfies $\left|K^{2}\right|<7$ for all 3-element subsets $K$ if and only if $G=S_{3}$. Also groups with the small cubing property on 2-sets are investigated.
\end{abstract}

\section{INTRODUCTION}

The problems considered in this paper come from a class of problems suggested by the second author, namely problems concerning the size of $K^{m}=\left\{a_{1} a_{2} \ldots a_{m} \mid a_{i} \in\right.$ $K$ for $1 \leqslant i \leqslant m\}$ for a $k$-element subset $K$ of a group $G$ as a function of $k$ and $m$. Several problems of this type have been considered in the literature. For example if $G$ is the additive group of integers and $K$ is a set of $k$ integers then (writing $K+K$ for $K^{2}$ )

$$
2 k-1 \leqslant|K+K| \leqslant k(k+1) / 2
$$

and it was shown in [7], see also [10], that $|K+K|=2 k-1$ if and only if $K$ is an arithmetic progression. Also, in [7], the structure of $K$ was determined if $|K+K|<c k$ for some constant $c$ and $k$ sufficiently large. More generally if $G$ is any torsion free

Received 3 December 1990

We wish to thank George Glauberman for suggesting a simplification of the proof of Lemma 5.4 (which is used to prove Theorem 5.1).

Copyright Clearance Centre, Inc. Serial-fee code: 0004-9729/91 SA2.00+0.00. 
group then (see [14], [10, Theorem 3] and [5]), $\left|K^{2}\right| \geqslant 2 k-1$ and equality holds if and only if $K$ is a progression, that is $K=\left\{a, a c, \ldots, a c^{k-1}\right\}=\left\{b, d b, \ldots, d^{k-1} b\right\}$ for some $a, b, c, d \in G$ with $c \neq 1, d \neq 1$. On the other hand if $G$ is not torsion free then it is possible for $\left|K^{2}\right|$ to be less than $2 k-1$. Trivially if $G$ is a finite group and $K=G$ then $K^{2}=K$. In [8] the second author showed that $\left|K^{2}\right|<3|K| / 2$ for some finite subset $K$ of a group $G$ if and only if either $K^{2}=\langle K\rangle$ (the group generated by $K$ ) or $K \subseteq g N$ for some $g \in G$ and some normal subgroup $N$ of $G$ of order $\left|K^{2}\right|$.

Let us say that a group $G$ has the small squaring property on $k$-sets if $\left|K^{2}\right|<k^{2}$ for all $k$-element subsets $K$. In order to gain an understanding of the structural restrictions placed on a group by the small squaring property on $k$-sets for a fixed $k$, the second author ([9]; see also [4]) investigated this property for 2-element subsets and showed that a finite group has the small squaring property on 2-sets if and only if it is a Dedekind group, that is, either it is abelian or the direct product of an elementary abelian 2-group and the quaternion group $Q_{8}$ of order 8. Recently Neumann [17] has shown that a group $G$ has the small squaring property on $k$-sets if and only if $G$ has normal subgroups $M, N$ with $1 \leqslant M<N \leqslant G$ such that $|M|$ and $|G / N|$ are bounded above by a function of $k$, and $N / M$ is abelian, that is $G$ is "finite by abelian by finite". In the present paper we consider finite groups with the small squaring property on 3-sets and obtain an almost complete characterisation, namely we obtain a characterisation of all examples except those of exponent 4 . The first main result is the following theorem which is proved in Section 2.

Theorem 1. Let $G$ be a finite group such that $\left|K^{2}\right|<9$ for all 3-element subsets $K$ of $G$. Then one of the following is true.

(a) The group $G$ is abelian.

(b) The group $G$ is a nonabelian 2-group.

(c) The group $G=T P$ where $T$ is a nontrivial normal abelian odd order subgroup and $P$ is a nontrivial 2-group. Further the subgroup $Q$ of $P$ which centralises $T$ has index 2 in $P$ and each element of $P-Q$ inverts $T$, and either

(i) $P$ is abelian and $Q$ is elementary abelian, or

(ii) $P$ is nonabelian, $Q$ is abelian, and each element of $P-Q$ inverts $Q$.

Conversely any group satisfying (a) or (c) has the small squaring property on 3-sets.

In Section 3 nonabelian 2-groups with the small squaring property on 3-sets are investigated. They are shown (Theorem 2) to be similar to dihedral or generalised quaternion groups unless they have exponent 4 , derived length 2 , and a very restrictive structure on the centre and derived quotient. We obtain a complete characterisation of 
all examples of exponent at least 8 .

If we impose an even stronger restriction on the orders of the squares of 3-element subsets then we obtain the following result. It will be proved in Section 4.

Theorem 3. Let $G$ be a finite nonabelian group. Then $G$ has the property that $\left|K^{2}\right|<7$ for all 3-element subsets $K$ of $G$ if and only if $G=S_{3}$.

In Section 5 we consider finite groups with the small cubing property on 2-sets. Our main result, Theorem 5.1 , is fairly technical but is sufficiently strong to characterise all odd order examples:

THEOREM 4. Let $G$ be a group of odd order. Then $\left|K^{3}\right|<8$ for all 2-element subsets $K$ of $G$ if and only if either $G$ is abelian or $G$ is a nonabelian group of exponent 3.

This problem was first considered in an unpublished manuscript [3] of the first two authors where a weak version of Theorem 5.1 was proved. The version of Theorem 5.1 in [3] was improved in [6, Theorem 3] to give a characterisation of all examples of finite groups $G$ with the small cubing property on 2-sets except for the case where $G$ has a normal abelian 2-complement. Their result [6, Theorem 3] is rather technical for use in applications. We derive from our result, Theorem 5.1, the following characterisation of finite groups $G$ in which $\left|K^{3}\right|<6$ for all 2-element subsets $K$ of $G$.

Theorem 5. Let $G$ be a finite group. Then $\left|K^{3}\right|<6$ for all 2-element subsets $K$ of $G$ if and only if either $G$ is abelian or $G$ is a nonabelian 2-group satisfying one of

(a) $G=\langle a, H\rangle$ where $H$ is an abelian subgroup of index 2 in $G$ and of exponent 4 , and $a$ has order 2 and inverts $H$.

(b) The Frattini subgroup $\Phi(G)$ of $G$ has order 2 .

\section{NONABELIAN GROUPS OTHER THAN 2-GROUPS WITH SMALL SQUARING ON} 3-SETS

Suppose that $G$ is a finite nonabelian group which is not a 2 -group and which has the small squaring property on 3-sets. We shall show that $G$ satisfies part (c) of Theorem 1. Note that each subgroup and quotient group of $G$ has the small squaring property on 3-sets. First we consider odd ordered Sylow subgroups of $G$.

LEMMA 2.1. Each Sylow subgroup of $G$ of odd order is abelian.

Proof: Suppose that $P$ is a nonabelian $p$-subgroup of $G$ for some odd prime $p$. Then $P / Z(P)$, where $Z(P)$ is the centre of $P$, is not cyclic and so $P$ has distinct maximal subgroups $M$ and $N$ containing $Z(P)$ (see [12, 5.1]). Further $M$ and $N$ are normal subgroups of $P$. Let $Q=M \cap N$ and let $a \in M-Q$. Now $M$ and $N$ are 
generated by $M-Q$ and $N-Q$ respectively. Thus $\langle a, N-Q\rangle=\langle a, N\rangle=P$, so if $a$ centralised every element of $N-Q$ then $a$ would lie in the centre of $P$ which is not the case. So there is an element $b \in N-Q$ such that $a b \neq b a$. Let $K=\left\{a, a b, b^{2}\right\}$. Then $|K|=3$ and we claim that $K^{2}=\left\{a^{2}, a^{2} b, a b^{2}, a b a, a b a b, a b^{3}, b^{2} a, b^{2} a b, b^{4}\right\}$ has order 9 .

Note that since $M$ is normal in $P$, the coset of $M$ containing the word $w=$ $a^{i_{1}} b^{j_{1}} \ldots a^{i_{r}} b^{j_{r}}$ is the coset $b^{j} M$ where $j=\sum j_{k}$ and the coset of $N$ containing $w$ is $a^{i} N$ where $i=\sum i_{k}$.

So, for example, the only elements of $K^{2}$ in $M$ are $a^{2}, a b^{3}$ and $b^{2} a b$, the latter two lying in $M$ if and only if $b^{3} \in M$. Then as $a^{2} N$ is different from the coset of $N$ containing $a b^{3}$ or $b^{2} a b$ it follows that $a^{2}$ is not equal to any of the other words in $K^{2}$. Similarly, considering membership of $N$ we find that $b^{4}$ is not equal to any of the other words in $K^{2}$. Also the only elements of $K^{2}$ in $a^{2} N-M$ are $a^{2} b, a b a$, and $a b a b$ and clearly these are all distinct. Thus the only equalities among the expressions in $K^{2}$ must be between $a b^{2}, b^{2} a, a b^{3}$ and $b^{2} a b$. The first two of these lie in $b^{2} M$ and the last two are in $b^{3} M$ so the only possible equalities are $a b^{2}=b^{2} a$ and $a b^{3}=b^{2} a b$, each of which implies the other. However as $b$ has odd order $a b^{2}=b^{2} a$ is equivalent to $a b=b a$ which does not hold. Thus $\left|K^{2}\right|=9$ which is a contradiction.

Lemma 2.2. A finite group of odd order with the small squaring property on 3-sets is abelian.

Proof: Suppose that there is a finite nonabelian group of odd order with the small squaring property on 3 -sets and let $G$ be such a group with the least possible order. Then all proper subgroups of $G$ are abelian. By Lemma 2.1 it follows that $G$ is not nilpotent and so by a result of Schmidt, see $[18,9.1 .9]$ or $[11],|G|=p^{u} q^{v}$ where $p$ and $q$ are distinct primes and $G$ has a cyclic Sylow p-subgroup $P=\langle a\rangle$ which is not normal in $G$ and a normal Sylow $q$-subgroup $Q$. Since $G$ is nonabelian $a$ does not centralise $Q$, that is $a b \neq b a$ for some $b \in Q$. Suppose first that $|P|=p^{u}>3$ and let $K=\left\{b, a, b a^{2}\right\}$. We claim that $K^{2}=\left\{b^{2}, b^{2} a^{2}, b a, b a^{2} b, b a^{3}, b a^{2} b a^{2}, a^{2}, a b, a b a^{2}\right\}$ has order 9. As in the previous lemma $a^{i_{1}} b^{j_{1}} \ldots a^{i_{r}} b^{j_{r}} Q=a^{i} Q$ where $i=\sum i_{k}$. Thus the only element of $K^{2} \cap Q$ is $b^{2}$, the only elements of $K^{2}$ in $a Q$ are $a b$ and $b a$ and these are distinct, the only elements of $K^{2}$ in $a^{3} Q$ are $b a^{3}$ and $a b a^{2}$ and these are distinct, and the only element of $K^{2}$ in $a^{4} Q$ is $b a^{2} b a^{2}$. Thus the only possible equalities among the expressions in $K^{2}$ are between $b^{2} a^{2}, b a^{2} b$ and $a^{2}$ and here the possibilities are $b^{2} a^{2}=b a^{2} b$ or $b a^{2} b=a^{2}$. The former is equivalent to $b a^{2}=a^{2} b$ which implies $b a=a b$ (since $a$ has odd order) and so does not hold. The latter is equivalent to $a^{-2} b a^{2}=b^{-1}$ which implies $b a^{4}=a^{4} b$ and hence $b a=a b$, which again is not true. Thus $\left|K^{2}\right|=9$ which is a contradiction. 
Thus $|P|=p^{u}=3$. In this case let $K=\{a, b, a b\}$ so that

$$
K^{2}=\left\{a^{2}, a^{2} b, a b, a b a, a b^{2}, a b a b, b a, b^{2}, b a b\right\} .
$$

Again the only element of $K^{2}$ in $Q$ is $b^{2}$. The elements of $K^{2}$ in $a Q$ are $a b, a b^{2}, b a$ and $b a b$ and the only possible equality among these is $a b^{2}=b a$, that is $a^{-1} b a=b^{2}$, so that $b=a^{-3} b a^{3}=b^{8}$, that is $b^{7}=1$. In this case $\langle a, b\rangle$ is a nonabelian subgroup of $G$ of order 21 and hence $G=\langle a, b\rangle$, but it is easy to check that $\left|L^{2}\right|=9$ for $L=\left\{a, b^{2}, a b\right\}$ which is a contradiction. Thus $a b, a b^{2}, b a$ and $b a b$ are all distinct, and the only equalities among expressions in $K^{2}$ must be between the elements $a^{2}$, $a^{2} b, a b a$ and $a b a b$ of $K^{2} \cap a^{2} Q$. Here the only possibility is $a^{2}=a b a b$, that is $a^{-1} b a=b^{-1}$ which implies $b a^{2}=a^{2} b$ and hence $b a=a b$ which is not the case. This final contradiction completes the proof

Now let $P$ be a Sylow 2-subgroup of $G$. We show next that $G$ has a normal 2-complement.

Lemma 2.3. The group $G$ has a normal subgroup $T$ of odd order such that $G=T P$.

Proof: Suppose that there is a finite nonabelian group with the small squaring property on 3-sets which does not have a normal odd order subgroup of 2-power index, and let $G$ be such a group with minimum order. Then every proper subgroup of $G$ has an odd order normal subgroup of 2-power index and so by [13, IV.5.4], the Sylow 2-subgroup $P$ is normal in $G$ of exponent at most $4,|G / P|=q^{v}$ for some odd prime $q$ and a Sylow $q$-subgroup $Q$ of $G$ is cyclic, say $Q=\langle a\rangle$. Since $Q$ is not normal in $G$, there is an element $b \in P$ such that $a b \neq b a$. Let $K=\{a, b, a b\}$. We claim that $\left|K^{2}\right|=9$ contradicting the small squaring property of $G$. Now $K^{2} \cap a P=$ $\left\{a b, b a, b a b, a b^{2}\right\}$ and the only possible equality among these expressions is $b a=a b^{2}$, that is, $a^{-1} b a=b^{2}$. This implies that $b$ and $b^{2}$ have the same order which is not true. Also, $K^{2} \cap a^{2} P=\left\{a^{2}, a^{2} b, a b a, a b a b\right\}$ and the only possible equality among these expressions is $a^{2}=a b a b$, that is $a^{-1} b a=b^{-1}$ so that $b$ commutes with $a^{2}$ and hence with $a$ which is not the case. Thus $\left|K^{2}\right|=9$ and this contradiction completes the proof.

Lemma 2.4. The normal subgroup $T$ is abelian and is not centralised by $P$.

Proof: It follows from Lemma 2.2 that $T$ is abelian. Suppose that $P$ centralises $T$. Then $G=P \times T$ and as $G$ is not abelian, $P$ is not abelian. As in the proof of Lemma 2.1, $P$ has distinct maximal normal subgroups $M$ and $N$ containing $Z(P)$ and there are elements $a \in M-N$ and $b \in N-M$ such that $a b \neq b a$. Let $c \in T-\{1\}$ and consider $K=\left\{a, b c, a b c^{2}\right\}$. (Note that $c$ commutes with $a$ and $b$ and, if $w=a^{i_{1}} b^{j_{1}} \ldots a^{i_{r}} b^{j_{r}}$ then $w M=b^{j} M, w N=a^{i} N$ where $j=\sum j_{k}, i=\sum i_{k}$.) Then $K^{2} \cap(N \times T)=$ 
$\left\{b^{2} c^{2}\right\}, K^{2} \cap a(N \times T)=\left\{a b c, b a c, a b^{2} c^{3}, b a b c^{3}\right\}$ and these elements are all distinct since $a b \neq b a$ and $c$ has odd order, $K^{2} \cap a^{2}(N \times T)=\left\{a^{2} b c^{2}, a b a c^{2}, a^{2}, a b a b c^{4}\right\}$ and again these elements are all distinct. Thus $\left|K^{2}\right|=9$ which is a contradiction.

Lemma 2.5. The subgroup $P$ has a subgroup $Q$ of index 2 which centralises $T$ and every element of $P-Q$ inverts $T$.

Proof: Let $a \in P$ be an element which does not centralise $T$, so $a b \neq b a$ for some $b \in T$. First we show that $a^{2} b=b a^{2}$. This is true if $a^{2}=1$ so assume that $a^{2} \neq 1$ and consider $K=\left\{b, b a, b a^{2}\right\}$. Then $K^{2} \cap a T=\left\{b^{2} a, b a b\right\}$ has order $2, K^{2} \cap a^{3} T=\left\{b a b a^{2}, b a^{2} b a\right\}$ has order 2 , and $K^{2} \cap\left(T \cup a^{4} T\right)=\left\{b^{2}, b a^{2} b a^{2}\right\}$, $K^{2} \cap a^{2} T=\left\{b^{2} a^{2}, b a b a, b a^{2} b\right\}$. Since $\left|K^{2}\right|<9$ some pair of expressions in one of the latter two sets must be equal in $G$. The only possibilities are $b^{2}=b a^{2} b a^{2}$ with $a^{4}=1$, or $b^{2} a^{2}=b a^{2} b$. In either case $b a^{2}=a^{2} b$.

Now consider $K=\left\{b, b a, b^{2} a\right\}$. We have $K^{2} \cap a T=\left\{b^{2} a, b^{3} a, b a b, b^{2} a b\right\}$ and $K^{2} \cap\left(T \cup a^{2} T\right)=\left\{b^{2}, b a b a, b a b^{2} a, b^{2} a b a, b^{2} a b^{2} a\right\}$. Since $\left|K^{2}\right|<9$ some pair of expressions in one of these sets must be equal in $G$. If $a^{2} \neq 1$ then the only possibilities are $b^{3} a=b a b$ and $b a b a=b^{2} a b^{2} a$. The latter is equivalent to $a^{-1} b a=b^{-1}$. In the case of the former equality we have $a b a^{-1}=b^{2}$, and as $b a^{2}=a^{2} b$ we have $b=a^{2} b a^{-2}=b^{4}$. Thus $b^{3}=1$ and so $a b a^{-1}=b^{-1}$. In either case $a$ inverts $b$. If $a^{2}=1$ then apart from the above possibilities there is also the possible equality $b^{2}=b a b^{2} a$. This implies that $a b a=b^{2}$ and hence that $b=a^{2} b a^{2}=b^{4}$ whence $b^{3}=1$. Thus in all cases $a$ inverts $b$.

Thus, for each $x \in T, x^{a}=a^{-1} x a$ is either $x$ or $x^{-1}$, and for some $b \in T-\{1\}$, $b^{a}=b^{-1}$. Suppose there is an $x \in T=\{1\}$ which commutes with $a$. Then $(x b)^{a}=$ $x b^{-1}$ is either $x b$ or $(x b)^{-1}=x^{-1} b^{-1}$ (since $T$ is abelian). Neither of these is possible and hence $a$ inverts each element of $T$.

Thus each element of $P$ either centralises or inverts $T$ and so $P$ has a subgroup $Q$ of index 2 which centralises $T$.

At this point we distinguish two cases according as $P$ is abelian or not.

Lemma 2.6. Either $P$ is abelian or there are elements $a \in P-Q$ and $b \in Q$ such that $a b \neq b a$.

Proof: If $a \in P-Q$ centralises $Q$ then $a$ centralises $\langle a, Q\rangle=P$, that is $a \in$ $Z(P)$. Thus if each element of $P-Q$ centralised $Q$ then each element of $\langle P-Q\rangle=P$ would centralise $Q$ and hence would lie in $Z(P)$, that is $P$ would be abelian.

First we consider the case of $P$ abelian.

Lemma 2.7. (a) If $P$ is abelian then $Q$ is elementary abelian.

(b) Conversely suppose that $G=T P$ is a finite group with $T$ a nontrivial abelian odd order normal subgroup and $P$ an abelian 2-group such that the centraliser $Q=$ 
$C_{P}(T)$ of $T$ in $P$ is elementary abelian of index 2 in $P$ and $P$ inverts $T$. Then $G$ has the small squaring property on 3-sets.

Proof: (a) Suppose that $P$ is abelian and that $Q$ contains an element $b$ of order 4. Let $a \in P-Q$. By replacing $b$ by $b^{-1}$ if necessary we may assume that $a^{2} b \neq 1$. Let $c \in T-\{1\}$ and consider $K=\left\{a c, a b c^{2}, c\right\}$. (Recall that $b c=c b$ and $a c=c^{-1} a$.) Now $K^{2} \cap P$ consists of $(a c)^{2}=a^{2}, c a c=a,\left(a b c^{2}\right)^{2}=a^{2} b^{2}$, and also $a b c^{3}=a b$ if $c^{3}=1$, and these elements are all distinct. The elements of $K^{2}$ not in $T \times Q$ are $a c^{2}$, $a, a b c^{3}$, and $c\left(a b c^{2}\right)=a b c$ and these are all distinct. The elements of $K^{2} \cap(T \times Q)$ not in $P$ are $a c\left(a b c^{2}\right)=a^{2} b c, a b c^{2}(a c)=a^{2} b c^{-1}$, and $c^{2}$ and these are all distinct. Thus $\left|K^{2}\right|=9$ which is a contradiction. So $Q$ is elementary abelian.

(b) Let $G, T, P, Q$ be as stated and let $a \in P-Q$. Let $K$ be any 3-element subset of $G$. If $K$ contains at least two elements of $T \times Q$ then $\left|K^{2}\right|<9$ since $T \times Q$ is abelian. So assume that $K$ contains two elements of $G-(T \times Q)$ say $x=a b c$ and $x^{\prime}=a b^{\prime} c^{\prime}$ where $b, b^{\prime} \in Q$ and $c, c^{\prime} \in T$. Then $x^{2}=(a b c)^{2}=a^{2}$ since $c a=a c^{-1}$, and $b^{2}=1$, similarly $\left(x^{\prime}\right)^{2}=a^{2}$. Thus $\left|K^{2}\right|<9$ and so $G$ has the small squaring property on 3-sets.

Now we consider the case where $P$ is not abelian.

Lemma 2.8. If $P$ is not abelian then $Q$ is abelian and each element of $P-Q$ inverts $Q$.

Proof: By Lemma 2.6 there are elements $a \in P-Q$ and $b \in Q$ such that $a b \neq b a$. Consider $K=\{a, a b, c\}$ where $c \in T-\{1\}$. The elements of $K^{2}$ not in $T \cup P$ are $a c, c a, a b c, c a b$ and these are all distinct. The elements of $K^{2} \cap P$ are $a^{2}, a^{2} b, a b a$ and $a b a b$. Since $\left|K^{2}\right|<9$ at least two of these elements are equal and hence $a^{2}=a b a b$, that is $b^{a}=b^{-1}$. In particular $b^{2} \neq 1$. Thus $a$ centralises or inverts each element of $Q$. If $a$ inverts each element of $Q$ then, for $x, y \in Q$, $x^{-1} y^{-1}=(y x)^{-1}=(y x)^{a}=y^{a} x^{a}=y^{-1} x^{-1}$ and it follows that $Q$ is abelian. Also in this case each element of $P-Q$ is of the form $a x$ for some $x \in Q$, and $a x$ inverts $Q$. Thus we may assume that, for some $c \in Q, c^{a}=c \neq c^{-1}$. In particular $c \neq b, c \neq b^{-1}$ and $c^{2} \neq 1$. Now $(b c)^{a}=b^{a} c^{a}=b^{-1} c \neq b c$ and hence $(b c)^{a}=(b c)^{-1}$. It follows that $b c=\left(b^{-1} c\right)^{-1}$, that is $b$ inverts $c$. (In particular $b c \neq c b$.)

Now consider $K=\{a, b x, c y\}$ where $x, y$ are distinct nontrivial elements of $T$. The elements of $K^{2}$ not in $T \times Q$ are $a b x, a c y, b x a=a b^{-1} x^{-1}, c y a=a c y^{-1}$ and these are all distinct. The elements of $K^{2}$ in $T \times Q$ are $a^{2}, b^{2} x^{2}, c^{2} y^{2}, b c x y, c b x y$ and as $\left|K^{2}\right|<9$ at least two of these elements are equal. It follows that $x y=1$ and either $a^{2}=b c$ or $a^{2}=c b$. It follows that $c$ (equal to $b^{-1} a^{2}$ or $a^{2} b^{-1}$ ) commutes with $b$. This contradiction completes the proof.

Lemma 2.9. Let $G=T P$ be a finite group with $T$ a nontrivial abelian odd 
order normal subgroup and $P$ a 2-group such that the centraliser $Q=C_{P}(T)$ of $T$ in $P$ is abelian of index 2 in $P$ and $P$ inverts $T \times Q$. Then $G$ has the small squaring property on 3-sets.

Proof: Let $K$ be a subset of $G$ of order 3. If $K$ contains at least two elements of the abelian group $T \times Q$ then $\left|K^{2}\right|<9$. So assume that $K$ contains two elements not in $T \times Q$, say $x=a b c$ and $x^{\prime}=a b^{\prime} c^{\prime}$ where $a \in P-Q, b, b^{\prime} \in Q, c, c^{\prime} \in T$. Then $x^{2}=a^{2}=\left(x^{\prime}\right)^{2}$ and hence again $\left|K^{2}\right|<9$.

\section{NONABElian 2-GROUPS With THE SMALl SQUARING PROPERTY ON 3-SETS}

Here we investigate nonabelian 2-groups with the small squaring property on 3 sets. A complete classification is obtained of those with exponent at least 8 . First let us consider some examples.

Definition 3.1: (a) A 2-group $G$ is called a $D$-group if it is nonabelian and the subgroup $A=\left\langle x \mid x \in G, x^{2} \neq 1\right\rangle$ is a proper subgroup of $G$.

(b) A 2-group $G$ is called a $Q$-group if it is nonabelian and satisfies the following conditions.

(i) $G$ has a subgroup $A$ of index 2 such that each element of $G-A$ has order 4 .

(ii) If $x, y \in G-A$ then $x^{2}=y^{2}$ and $a^{x}=x^{-1} a x=a^{-1}$ for all $a \in A$.

The dihedral and generalised quaternion groups of order $2^{n} \geqslant 8$ are examples of $D$-groups and $Q$-groups respectively. All $D$-groups and $Q$-groups have the small squaring property on 3-sets.

LEMMA 3.2 . (a) If a 2 -group $G$ is a $D$-group then $A=\left\langle x \mid x^{2} \neq 1\right\rangle$ is an abelian subgroup of index 2, each element of $G-A$ inverts $A$, and $G$ has the small squaring property on 3-sets.

(b) If a 2-group $G$ is a $Q$-group then $G$ has the small squaring property on 3-sets and the subgroup $A$ of 3.1 (b)(i) is abelian.

Proof: (a) Since $G=\langle G-A\rangle$ and $G$ is nonabelian there are non-commuting elements $x, y$ in $G-A$ and by the definition of $A, x^{2}=y^{2}=1$. Suppose that $|G: A|>2$. Then we may choose $x$ and $y$ so that $x y \neq y x$ and also $x A \neq y A$. Since $A$ is normal in $G$, the four cosets $A, x A, y A$ and $x y A=A x y$ are all distinct. Thus $x y \in G-A$ and hence has order 2 . This implies that $x y=y x$ which is a contradiction. Thus $A$ has index 2 in $G$. Now if $a \in A$ then $x a \in G-A$ and so $(x a)^{2}=1$, that is $a^{x}=a^{-1}$. So $x$ inverts every element of $A$ and it follows that $A$ is abelian. Let $K$ be a 3-element subset of $G$. If $|K \cap A| \geqslant 2$ then $\left|K^{2}\right|<9$ since $A$ is abelian while if $K$ contains two elements $x, y$ of $G-A$ then $x^{2}=y^{2}=1$ and again $\left|K^{2}\right|<9$. 
(b) Since an element of $G-A$ inverts $A, A$ is abelian. Then if $K$ is a 3-element subset of $G,\left|K^{2}\right|<9$ when $|K \cap A| \geqslant 2$ since $A$ is abelian, and $\left|K^{2}\right|<9$ when $K$ contains two elements $x, y$ of $G-A$ since $x^{2}=y^{2}$.

The main result which will be proved in this section is the following theorem.

TheOREM 2. Let $G$ be a nonabelian 2-group such that $\left|K^{2}\right|<9$ for all 3element subsets $K$ of $G$. Then either

(a) $G$ is a $D$-group or a $Q$-group, or

(b) $G$ has exponent 4 and derived length 2. Further each of the centre $Z(G)$ and the derived quotient $G / G^{\prime}$ is either elementary abelian or the product of an elementary abelian group and a cyclic group of order 4 .

Conversely each 2-group which is a $D$-group or a $Q$-group has the small squaring property on 3-sets.

The problem of determining all exponent 4 examples is open. There are certainly examples which are not $D$-groups or $Q$-groups and this is demonstrated in the following result.

PROPOSITION 3.3. Let $G$ be a nonabelian 2-group which is minimal (by inclusion) such that $\left|K^{2}\right|<9$ for all 3-element subsets $K$. Then $G$ is $D_{8}, Q_{8}$ or $T=\langle a, b| a^{4}=b^{4}=1, b^{-1} a b=a^{-1}$ ) (and all of these groups have the small squaring property on 3-sets).

Proof: If $|G|=8$ then $G$ is $D_{8}$ or $Q_{8}$ which have the small squaring property by Lemma 3.2, so assume that $|G| \geqslant 16$. Now $G$ is a minimal nonabelian 2-group since all subgroups of $G$ have the small squaring property. Then, see [13, p.309], either

$$
G=G_{1}=\left\langle a, b \mid a^{2^{\alpha}}=b^{2^{\beta}}=1, b^{-1} a b=a^{1+2^{\alpha-1}}\right\rangle
$$

with $\alpha \geqslant 2$, and $|G|=2^{\alpha+\beta}$, or

$$
G=G_{2}=\left\langle a, b \mid a^{2^{\alpha}}=b^{2^{\beta}}=1,[a, b]^{2}=1\right\rangle,
$$

with say $\alpha \geqslant 2$ and $|G|=2^{\alpha+\beta+1}$.

Suppose $G=G_{1}$. Then $Z(G)=\left\langle a^{2}, b^{2}\right\rangle$ has index 4. If $\alpha=3$ then $\left|K^{2}\right|=9$ for $K=\{a, b, b a\}$, so $\alpha=2$ and hence $\beta \geqslant 2$. If $\beta \geqslant 3$ then $\left|K^{2}\right|=9$ for $K=\left\{a, b, a b^{3}\right\}$, so $\beta=2$ and $G=T$. It can be checked that $T$ has the small squaring property on 3 -sets.

Now let $G=G_{2}$. Then $Z(G)=\left\langle a^{2}, b^{2},[a, b]\right\rangle$ again has index 4 , and $\left|K^{2}\right|=9$ if $K=\{a, b, b a\}$. Thus $G_{2}$ does not have the small squaring condition.

This result is very important when investigating nonabelian 2-groups $G$ with the small squaring property on 3 -sets as the small squaring property is inherited by subgroups and quotient groups. For example if $H$ is a minimal nonabelian subgroup of 
$G$, then by Proposition 3.3, $H$ is isomorphic to $D_{8}, Q_{8}$, or the group $T$ defined there. Also if $A$ is a cyclic subgroup of $Z(G)$ then $H A$ is a direct product or a central product of $H$ and $A$, and $H A$ has the small squaring property on 3-sets. We shall need some information about such products.

PROPOSITION 3.4. (a) None of the central products $Z_{8} * D_{8}$ and $Z_{8} * Q_{8}$ and the direct product $Z_{8} \times Q_{8}$ has the small squaring property on 3-sets.

(b) The direct product $Z_{4} \times L$ of a cyclic group of order 4 and a nonabelian 2-group $L$ has the small squaring property on 3-sets if and only if $L=Q_{\mathrm{B}} \times E$, where $E$ is an elementary abelian 2-group or $E=1$.

(c) A central product of a cyclic group $Z_{4}=\langle c\rangle$ and the group $T=\langle a, b| a^{4}=$ $\left.b^{4}=1, b^{-1} a b=a^{-1}\right\rangle$ has the small squaring property on 3-sets if and only if $c^{2}=a^{2} b^{2}$. $A$ central product of the abelian group $Z_{4} \times Z_{4}=\langle c\rangle \times\langle d\rangle$ and $T$ does not have the small squaring property on 3-sets.

Proof: (a) This is proved by showing that $\left|K^{2}\right|=9$ for the following sets $K$. For $Z_{8} * D_{8}=\left\langle c, a, b \mid a^{4}=b^{2}=1, c^{4}=a^{2}, c a=a c, c b=b c, b a=a^{3} b\right\rangle$ let $K\{a, b, a b c\}$, and for $Z_{8} \times Q_{8}=\langle c\rangle \times\left\langle a, b \mid a^{4}=1, a^{2}=b^{2}, b a=a^{3} b\right\rangle$ and $Z_{8} * Q_{8}=\langle c, a, b| a^{4}=$ $\left.1, c^{4}=a^{2}=b^{2}, c a=a c, c b=b c, b a=a^{3} b\right\rangle$ let $K=\left\{a, b c, a b c^{3}\right\}$.

(b) Suppose that $Z_{4} \times L=\langle c\rangle \times L$ has the small squaring property on 3-sets but that $L$ is not of the required form. Then by Freiman [9], there is a subset $K_{0}=\{a, b\}$ of $L$ such that $\left|K_{0}^{2}\right|=4$. Since for $K=\{a, b, a b c\}$ we must have $\left|K^{2}\right|<9$, it follows that $b(a b c)=(a b c) a$ and hence that $a=(a b)^{-1} b(a b)$. Thus $L_{0}=\langle a, b\rangle$ is a finite nonabelian 2-group in which $a$ and $b$ are conjugate. This is impossible. (For suppose that $L_{0}=\langle a, b\rangle$ has least order among finite nonabelian 2-groups with the generators $a$ and $b$ conjugate in $L_{0}$. Then $L_{0} / Z\left(L_{0}\right)=\left\langle a Z\left(L_{0}\right), b Z\left(L_{0}\right)\right\rangle$ has its generators $a Z\left(L_{0}\right)$ and $b Z\left(L_{0}\right)$ conjugate, so by minimality $L_{0} / Z\left(L_{0}\right)$ is abelian. As $a Z\left(L_{0}\right)$ and $b Z\left(L_{0}\right)$ are conjugate in $L_{0} / Z\left(L_{0}\right)$ they are equal and hence $L_{0} / z\left(L_{0}\right)$ is cyclic. Thus $L_{0}$ is abelian, which is a contradiction.) Thus $L$ is of the required form.

Conversely consider $H=Z_{4} \times L=\langle c\rangle \times L$ where $L=Q \times E$, with $Q=\langle a, b| a^{4}=$ $\left.1, a^{2}=b^{2}, b a=a^{3} b\right\rangle$ and $E$ an elementary abelian 2-group. Let $K$ be a 3-element subset of $\boldsymbol{H}$. If $K \cap L$ contains distinct elements $x$ and $x^{\prime}$ then by $[9],\left|\left\{x, x^{\prime}\right\}^{2}\right|<4$ and so $\left|K^{2}\right|<9$. Similarly if $K \cap\left(c L \cup c^{3} L\right)$ contains distinct elements $c^{6} x$ and $c^{6^{\prime}} x^{\prime}$, where $\delta, \delta^{\prime} \in\{1,3\}$ and $x, x^{\prime} \in L$, then $\left\{c^{\delta} x, c^{\delta^{\prime}} x^{\prime}\right\}^{2}=c^{2}\left\{x, x^{\prime}\right\}^{2}$ has size at most 3 and so $\left|K^{2}\right|<9$. Again if $K \cap c^{2} L$ contains distinct elements $c^{2} x$ and $c^{2} x^{\prime}$, where $x$, $x^{\prime} \in L$ then $\left\{c^{2} x, c^{2} x^{\prime}\right\}^{2}=\left\{x, x^{\prime}\right\}^{2}$ has size at most 3 and $\left|K^{2}\right|<9$. The remaining case to be considered is $K=\left\{x, c^{2} x^{\prime}, c^{6} x^{\prime \prime}\right\}$ where $x, x^{\prime}, x^{\prime \prime} \in L$ and $\delta$ is 1 or 3 . If any one of $x, x^{\prime}, x^{\prime \prime}$ lies in $Z(L)$ then $\left|K^{2}\right|<9$. So we may assume that all of them lie in $L-Z(L)$, but then $x^{2}=x^{\prime 2}$ and again $\left|K^{2}\right|<9$. Thus $H$ has the small 
squaring property on 3 -sets.

(c) By (b), $T \cap\langle c\rangle \neq 1$. Hence $c^{2} \in\left\{a^{2}, b^{2}, a^{2} b^{2}\right\}$. Take $K=\{a, b, a b c\}$. If $\left|K^{2}\right|<, 9$ then $c^{2} \neq a^{2}, b^{2}$. Thus $c^{2}=a^{2} b^{2}$, and in this case it is easy to check that $T *\langle c\rangle$ with $c^{2}=a^{2} b^{2}$ has the small squaring property on 3-sets. Finally suppose that some central product $G=T *(\langle c\rangle \times\langle d\rangle)$ has the small squaring property. Then $T *\langle c\rangle$ and $T *\langle d\rangle$ have the small squaring property also, and hence $c^{2}=a^{2} b^{2}=d^{2}$. Therefore $c^{2}=d^{2} \in\langle c\rangle \cap\langle d\rangle$, which is a contradiction.

This Proposition yields the result claimed for the structure of $Z(G)$ in Theorem 3.

THEOREM 3.5. Let $G$ be a nonabelian 2-group with the small squaring property on 3-sets. Then $Z(G)$ is either $E$ or $E \times Z_{4}$, where $E$ is elementary abelian (or trivial).

Proof: Let $H$ be a minimal nonabelian subgroup of $G$. Suppose that $Z(G)$ is not elementary abelian and let $A=\langle a\rangle$ be a cyclic direct summand of $Z(G)$ of maximal order. By Propositions 3.3 and 3.4 applied to $H$ and $H A$ it follows that $|A|=4$ and $H$ is $D_{8}$ or $Q_{8}$ or $T$. Now suppose that $Z(G)$ has a subgroup $A \times B \cong Z_{4} \times Z_{4}$. If $H \cap(A \times B)=1$ then $H(A \times B)=(H \times A) \times B$ cannot have the small squaring property on 3-sets by Proposition 3.4(b), which is a contradiction. Thus we may assume that $B \cap A \neq\{1\}$. Suppose first that $H$ is $D_{8}$ or $Q_{8}$. Then $Z(H A)=A$, so that $H A \cap B=1$. Hence $H(A \times B)=(H A) \times B$ and this does not have the small squaring property on 3-sets by Proposisiton 3.4, a contradiction. Now suppose that $H=T=\left\langle a, b \mid a^{4}=b^{4}=1, b^{-1} a b=a^{-1}\right\rangle$. Then $H(A \times B)$ does not have the small squaring property on 3-sets by Proposition 3.4(c). Thus $Z(G) \cong E \times A$ with $E$ elementary abelian or trivial.

Next we investigate the structure of the derived quotient $G / G^{\prime}$ of a nonabelian 2-group $G$ with the small squaring property on 3 -sets. Let

$$
G / G^{\prime}=\left\langle a G^{\prime}\right\rangle \times\left\langle c_{1} G^{\prime}\right\rangle \times \ldots \times\left\langle c_{n} G^{\prime}\right\rangle
$$

where $O\left(a G^{\prime}\right) \geqslant O\left(c_{1} G^{\prime}\right) \geqslant \ldots . . \geqslant O\left(c_{n} G^{\prime}\right)$, (where $O(g)$ denotes the order of $g$ ). To obtain restrictions on the orders of the cyclic direct summands we shall examine certain sections of $G$, for example certain quotients of $\left\langle a, c_{i}\right\rangle$ for some $i$. We shall need the following lemma which allows us to make use of Proposition 3.3 again.

LEMMA 3.6. Let $L$ be a nonabelian 2-group with derived group $L^{\prime}$ of order 2 . Then $L / Z(L)$ has exponent 2. In particular if $L$ has a generating set of size 2 then $L$ is a minimal nonabelian group.

Proof: Since $\left|L^{\prime}\right|=2$ it follows that $L^{\prime} \subseteq Z(L)$. Let $x, y \in L$. Then $1=$ $[x, y]^{2}=x^{-1}[x, y] y^{-1} x y=\left[x^{2}, y\right]$. Hence $x^{2} \in Z(L)$ for all $x \in L$, that is $L / Z(L)$ has exponent 2. Now let $L=\langle a, b\rangle$. Then $L / Z(L)=\langle a Z(L), b Z(L))$ is elementary abelian of order 4 . If $B$ is any subgroup of $L$ of index 2 then $H$ contains $Z(L)$. (For if not then 
$L=H Z(L)$, so $H$ contains $a z$ and $b z^{\prime}$ for some $z, z^{\prime}$ in $Z(L)$ and hence $H$ contains $\left\langle\left[a z, b z^{\prime}\right]=[a, b]\right\rangle=L^{\prime}$. Then $H / L^{\prime}$ is a subgroup of $L / L^{\prime}=\left\langle a L^{\prime}\right\rangle \times\left\langle b L^{\prime}\right\rangle$ of index 2 and so $H / L^{\prime}$ contains $Z(L) / L^{\prime}=\left\langle a^{2} L^{\prime}\right\rangle \times\left\langle b^{2} L^{\prime}\right\rangle$ which contradicts $L=H Z(L)$.) Then since $|H: Z(L)|=2$ it follows that $H$ is abelian, and hence that $L$ is a minimal nonabelian group.

THEOREM 3.7. Let $G$ be a nonabelian 2-group with the small squaring property on 3-sets. Then $G / G^{\prime}$ is either $E$ or $Z_{4} \times E$ where $E$ is elementary abelian (or trivial).

Proof: We use the notation introduced before Lemma 3.6. Note that, since $G^{\prime} \subseteq$ $\Phi(G)$, we have $G=\left\langle a, c_{1}, \ldots, c_{n}\right\rangle$. Suppose first that $O\left(a G^{\prime}\right) \geqslant 8$. Then, by Theorem $3.5, a \notin Z(G)$, and so for some $i=1, \ldots, n, H=\left\langle a, c_{i}\right\rangle$ is nonabelian. Let $R$ be a subgroup of $H^{\prime}$ of index 2 which is normal in $H$. By Lemma 3.6, $L=H / R$ is a minimal nonabelian group. Since $L$ has exponent at least 8 it follows from Proposition 3.3 that $L$ does not have the small squaring property on 3-sets, which is a contradiction. Thus $O\left(a G^{\prime}\right)=4$.

Next suppose that $a \in Z(G)$. Then by Theorem 3.5, $a$ has order 4 , and it follows that $G \cong\langle a\rangle \times\left\langle c_{1}, \ldots, c_{n}\right\rangle$. By Proposition 3.4(b), $G \cong\langle a\rangle \times Q_{8} \times E$ where $E$ is elementary abelian, and so $G / G^{\prime}$ is the direct product of $Z_{4}$ and an elementary abelian group. Thus we may suppose that $a \notin Z(G)$. Suppose that $c_{1} G^{\prime}$ has order 4 . Consider $\left\langle c_{1}, \ldots, c_{n}\right\rangle$. If $a$ centralised every element $c$ of this group for which $O\left(c G^{\prime}\right)=4$ then, since the elements of order 4 in the abelian group $G / G^{\prime}$ generate $G / G^{\prime}$, it follows that $a$ would centralise $\left\langle c_{1}, \ldots, c_{n}\right\rangle$ and hence $a$ would lie in $Z(G)$. Thus we may assume that $a c_{1} \neq c_{1} a$. Let $H=\left\langle a, c_{1}\right\rangle$ and let $R$ be a subgroup of $H^{\prime}$, of index 2 in $H^{\prime}$, which is normal in $H$. Then, by Lemma 3.6, $H / R$ is minimal nonabelian, and by Proposition 3.3, $H / R$ does not have the small squaring property on 3 -sets, which is a contradiction. Thus $G / G^{\prime}$ has the required form.

Our next task is to examine examples of large exponent. First we deal with those containing an abelian subgroup of index 2 .

Proposition 3.8. Let $G$ be a nonabelian 2-group with the small squaring property on 3-sets. If $G$ has an abelian subgroup $A$ of index 2 with exponent $\exp (A)$ at least 8 then $G$ is a $D$-group or a $Q$-group.

Proof: Let $C=\langle c\rangle$ be a cyclic subgroup of $A$ of order $\exp (A)=2^{n}$ say, and let $b \in G-A$. Then $G=\langle b, A\rangle=A \cup b A$. Also, by Theorem 3.5, $c \notin Z(G)$ and hence $b c \neq c b$. Assume first that $b^{2}, c^{2}$ and $(b c)^{2}$ are all distinct, and consider $K=\{b, c, b c\}$. Now $K^{2} \cap b A=\left\{b c, c b, c b c, b c^{2}\right\}$ and these elements are all distinct (for the only possible equality is $c b=b c^{2}$ which implies $b^{-1} c b=c^{2}$ whence $c$ and $c^{2}$ have the same order, a contradiction). The set $K^{2} \cap A=\left\{b^{2}, b^{2} c,(b c)^{2}, c^{2}, b c b\right\}$ and the only possible equalities between these elements are $b^{2} c=c^{2}$ and $c^{2}=b c b$. If 
$b^{2} c=c^{2}$ then $c=b^{2}$ whence $C_{G}(c)$ contains $\langle b, A\rangle=G$, that is $c \in Z(G)$ which is a contradiction. If $c^{2}=b c b$ then $c^{3}=(b c)^{2}$ whence $C_{G}\left(c^{3}\right)$ contains $\langle A, b c\rangle=G$, that is $c^{3}$ and hence $c$ lie in $Z(G)$, again a contradiction. Thus $\left|K^{2}\right|=9$ which is not the case. So for any element $c$ of maximal order in $A$, and any $b \in G-A$, the elements $b^{2}, c^{2}$ and $(b c)^{2}$ are not all distinct.

Suppose that $b^{2}=c^{2}$ for some such $b, c$. Then $b$ has order $2^{n}$. Let $L=\langle b, c\rangle$. Then $L$ is nonabelian (recall $b c \neq c b$ ) and $c^{2}$ lies in its centre. So by Theorem 3.5, $c^{2}$ has order at most 4. Thus $\exp (A)=2^{n}=8$. We claim that $b$ inverts $c$. For $d=b^{3} \in G-A$ and $d^{2}=b^{6}=c^{6} \neq c^{2}$ so either $d^{2}=(d c)^{2}$ or $c^{2}=(d c)^{2}$. In the latter case, $c=d c d=d(d c d) d=d^{2} c d^{2}=c^{6+2+6}=c^{6}$ which is a contradiction. Thus $d^{2}=(d c)^{2}$, that is $d^{-1} c d=c^{-1}$ and it follows that $b=d^{3}$ inverts $c$. Similarly, if $(b c)^{2}=c^{2}$ then $\exp (A)=8$, and $b c$, and hence $b$ inverts $c$. Finally if $c^{2}$ is not equal to $b^{2}$ or $(b c)^{2}$ then we must have $b^{2}=(b c)^{2}$, and again $b^{-1} c b=c^{-1}$.

Thus we have shown that, for each $b \in G-A$ and each $c \in A$ of order $2^{n}, b$ inverts $c$, and if either $b^{2}=c^{2}$ or $(b c)^{2}$ for some such $b, c$, then $A$ has exponent 8 . Now, as $A$ is generated by its elements of order $2^{n}$, each element $b \in G-A$ inverts each element of $A$. In particular, for $c$ of order $2^{n}$ in $A, b^{2} c^{-1} \in A$ and so $b$ inverts $c$ and $b^{2} c^{-1}$. Thus we have $b^{2} c^{-1}=b^{-1} b^{2}\left(b c^{-1}\right)=b^{-1} b^{2} c b=\left(b^{2} c\right)^{-1}=c^{-1} b^{-2}=b^{-2} c^{-1}$ (as $A$ is abelian), and hence $b^{4}=1$. Thus each element $b$ of $G-A$ has order at most 4. Suppose that some $b \in G-A$ has order 2. Then, for each $a \in A$ we have $(b a)^{2}=(b a b) a=a^{-1} a=1$, that is each element of $G-A$ has order 2 . In this case $G$ is a $D$-group. Thus we may assume that each element of $G-A$ has order 4 . If $b, d \in G-A$ then $d=b a$ for some $a \in A$ and so $d^{2}=b(a b) a=b b a^{-1} a=b^{2}$. Thus $G$ is a $Q$-group.

Now we complete the classification for the case of large exponent.

ThEOREM 3.9. Let $G$ be a nonabelian 2-group of exponent at least 8 with the small squaring property on 3-sets. Then $G$ is a $D$-group or a $Q$-group.

Proof: We prove this Proposition by induction on $|G|$. Now $|G| \geqslant 16$ and if $|G|=16$ then the result follows from Proposition 3.8. So assume that the result is true for groups of order less than $|G|$. Let $G$ have exponent $2^{n} \geqslant 8$ and let $C=\langle c\rangle$ be a cyclic subgroup of order $2^{n}$ in $G$. By Proposition 3.8 we may assume that $G$ does not contain an abelian subgroup of index 2 and exponent $2^{n}$. Let $M$ be a subgroup of $G$ containing $C$ with $|G: M|=2$. Then $M$ is not abelian, and by induction $M$ is a $D$-group or a $Q$-group. It follows from Lemma 3.2 that $M$ has a characteristic subgroup $A$ of index 2 which is abelian. Thus $A$ is normal in $G$ of index 4 .

Suppose that $G / A$ is cyclic, say $G / A=\langle a A\rangle$. By Definition 3.1 and Lemma 3.2, $a^{2}$ inverts $A$ and $a^{2}$ has order at most 4. Let $K=\{a, c, a c\}$. Then $K^{2} \cap A=\left\{c^{2}\right\}$. 
In the set $K^{2} \cap a A=\left\{a c, c a, a c^{2}, c a c\right\}$ the only possible equality is $c a=a c^{2}$ which implies $a^{-1} c a=c^{2}$. This is impossible since $c$ and $c^{2}$ have different orders. Thus $\left|K^{2} \cap a A\right|=4$. Also $K^{2} \cap a^{2} A=\left\{a^{2},(a c)^{2}, a^{2} c, a c a\right\}$ and the only possible equality between these elements is $a^{2}=(a c)^{2}$, that is $a^{-1} c a=c^{-1}$. However this would imply that $a^{2}$ centralised $c$ which is not the case. Hence $\left|K^{2}\right|=9$ which is a contradiction. Thus $G / A$ is elementary abelian of order 4 . Let $M_{i}, i=1,2,3$, be the subgroups of index 2 in $G$ containing $A$. As above each $M_{i}$ is a $D$-group or a $Q$-group. Let $M_{i}=\left\langle a_{i}, A\right\rangle$ for $i=1,2,3$. Suppose that $A$ is not cyclic. Then $A-C$ contains an involution $x$ say, and as $a_{i}$ inverts $A, a_{i} x=x a_{i}$ for each $i=1,2,3$. Hence $x \in Z(G)$. Now $G /\langle x\rangle$ has exponent $2^{n}$ (as $C \simeq C\langle x\rangle /\langle x\rangle$ ), is nonabelian, and is not a $D$-group or a $Q$-group (since $A /\langle x\rangle$ is a maximal abelian subgroup of $G /\langle x\rangle$ and it has index 4 and order at least $\left.2^{n}\right)$. This contradicts the inductive assumptions. Thus $A=\langle c\rangle$ is cyclic. If $M_{i}$ is a $D$-group then by definition $a_{i}^{2}=1$ and $M_{i}$ is dihedral of order $2^{n+1}$. If $M_{i}$ is a $Q$-group then $a_{i}$ has order 4 and $M_{i}$ is a generalised quaternion group of order $2^{n+1}$. Thus each of the $M_{i}$ is a nonabelian 2-group of maximal class (see [12, 5.4.5]), and $G$ itself is not of maximal class. Hence the subgroup $A$ is contained in exactly 3 subgroups of $G$ of maximal class and order $2^{n+1}$, whereas it was shown in [2] that a proper subgroup $A$ of a 2-group $G$, where $G$ is not of maximal class, is such that $A$ is contained in an even number of subgroups of maximal class and given order $2^{r}>|A|$. This contradiction completes the proof of Theorem 3.9.

Finally we obtain a bound on the derived length. Note that each $D$-group and $Q$-group has derived length 2 .

THEOREM 3.10. Let $G$ be a nonabelian 2-group with the small squaring property on 3-sets. Then $G$ has derived length 2 .

Proof: We prove this result by induction on $|G|$. It is certainly true for $|G|=8$ so we assume that $|G|>8$ and that the result is true for groups of order less than $|G|$. We may assume that $G$ has exponent 4. Suppose that $G^{\prime \prime} \neq 1$. Then $G$ has a normal subgroup $R$ which is elementary abelian of order 4 (see [13, III] or [1]). Suppose that $R$ is contained in $Z(G)$, and let $M_{1}, M_{2}$ be distinct subgroups of $R$ of order 2. Then by induction $\left(G / M_{1}\right)^{\prime \prime}=1$ and $\left(G / M_{2}\right)^{\prime \prime}=1$ and hence $G^{\prime \prime} \leqslant M_{1} \cap M_{2}=1$, which is a contradiction. Hence $R \nless Z(G)$. Now $R \neq \Phi(G)$, for it was shown in [15] that a 2-group $G$ with Frattini subgroup $\Phi(G)=Z_{2} \times Z_{2}$ is such that $\Phi(G) \leqslant Z(G)$. If $\Phi(G) \subset R$ then $|\Phi(G)|=2$ and $G^{\prime \prime}=1$. Hence $R \ngtr \Phi(G)$, and so $G / R$ has exponent 4. Also $G / R$ is nonabelian since $G^{\prime \prime} \neq 1$. Let $x \in G-R$ be such that $x^{2} \notin R$ and consider $L=\langle x, R\rangle$. Then $|L|=16$ and if $L$ were nonabelian it would be minimal nonabelian (note that $x^{2}$ centralises $R$ ) and by Proposition 3.3, $L \simeq T=\left\langle a, b \mid a^{4}=b^{4}=1, b^{-1} a b=a^{-1}\right\rangle$ which is not the case. Thus $L$ is abelian, that is $x$ centralises $R$. 
Now consider $A=\langle x \mid O(x R)=4\rangle$. Then $A$ contains $R$ and $|G: A| \leqslant 2$ (for $G / R$ is nonabelian, and if $A / R \neq G / R$ then the arguments in the proof of Lemma 3.2(a) show that $|G: A|=2$ ). Also $R$ centralises each element of $A$. If $G=A$ then $R \leqslant Z(G)$ which is not the case. Thus $|G: A|=2$, and so $G / R$ is a $D$-group. Also $C_{G}(R)=A$, and $G=\langle A, d\rangle$ for some $d$ such that $d^{2} \in R$. Now $d \notin C_{G}(R)$ and so $\langle d, R\rangle \simeq D_{8}$ (a nonabelian group of order 8 containing $Z_{2} \times Z_{2}$ ). Thus $\langle d, R\rangle-R$ contains an involution, and we may assume therefore that $d^{2}=1$. Let $a \in A$ be such that $a^{2} \notin R$ (that is $O(a R)=4)$. Let $b \in R-Z(\langle d, R\rangle)$, and let $c=d b d$. Then $Z(\langle d, R\rangle)=$ $\langle b c\rangle$. Since $G / R$ is a $D$-group it follows from Lemma 3.2 that $\operatorname{dad} R=a^{-1} R$. If $d a d=a^{-1} b$ then $a=d(d a d) d=d a^{-1} b d=(d a d)^{-1} d b d=\left(a^{-1} b\right)^{-1} c=b a c=a b c$ (since $a \in C_{G}(R)$ ), and hence $b c=1$ which is a contradiction. Similarly $d a d \neq a^{-1} c$. Hence dad is either $a^{-1}$ or $a^{-1} b c$.

Suppose that $\operatorname{dad}=a^{-1}$, and consider $K=\{a, d, a b d\}$. Then $K^{2}-A=$ $\left\{a d, d a, a^{2} b d, a b d a\right\}$. If $a d=a b d a$ then $d=b d a=d c a$, so $c a=1$, contradiction. If $d a=a^{2} b d$ then $a^{-1} d=a^{2} b d$ so that $a^{3}=b^{-1} \in R$, contradiction. If $a^{2} b d=a b d a=a b a^{-1} d$, again a contradiction as $a$ centralises $R$. Thus $\left|K^{2}-A\right|=4$. Also $K^{2} \cap A=\left\{a^{2}, d a b d=a^{-1} c, d^{2}=1, a b d^{2}=a b,(a b d)^{2}=b c\right\}$ has size 5 and so $\left|K^{2}\right|=9$ which is a contradiction.

Thus $d a d=a^{-1} b c$. Consider $K=\{a, d, b d\}$. Here $K \cap R=R, K^{2} \cap(A-R)=$ $\left\{a^{2}\right\}$, and $K-A=\{a d, d a, a b d, b d a\}$ has order 4. Thus $\left|K^{2}\right|=9$. This final contradiction completes the proof of Theorem 3.10.

The main theorem of this section, Theorem 2 , now follows immediately from Theorems $3.5,3.7,3.9$ and 3.10 .

\section{GROUPS IN WhICH $|K|<7$ FOR ALL $\left|K^{2}\right|=3$}

In this section we prove Theorem 3. Suppose that $G$ is a finite nonabelian group such that $\left|K^{2}\right|<7$ for all 3-element subsets $K$ of $G$. We shall prove that $G$ is isomorphic to $S_{3}$. Clearly $S_{3}$ has this property.

Suppose first that $G$ is a 2-group. Then, as $G$ is not abelian, $G / Z(G)$ is not cyclic and so $G$ has distinct maximal normal subgroups $M, N$ both containing $Z(G)$. Let $a \in M-N$. Then $a \notin Z(G)$ and so $a$ does not centralise $N=\langle N-M\rangle$, so there is an element $b \in N-M$ such that $a b \neq b a$. If $K=\{a, b, a b\}$ than $\left|K^{2}\right| \geqslant 7$ which is a contradiction. Thus $G$ is not a 2-group. By Theorem $1, G=T P$ where $T$ is an abelian normal odd order subgroup and $P$ is a nontrivial 2-group. Also $P$ contains an element $a$ which inverts $T$. Suppose that $|T|>3$ and let $b, c \in T-\{1\}$ be such that $c \neq b, c \neq b^{-1}$. Then for $K=\{a, b, c\}$, we have $\left|K^{2}\right| \geqslant 7$ which is a contradiction. Thus $T=\langle b\rangle$ is cyclic of order 3 . Also if $a^{2} \neq 1$ then for $K=\{a, b, a b\}$ we have 
$\left|K^{2}\right| \geqslant 7$. Thus $a^{2}=1$ and $\langle a, b\rangle \simeq S_{3}$. If $|P|>2$ then $P$ contains an element $c$ distinct from $a$ such that $c$ inverts $T$. As above $c^{2}=1$. Then for $K=\{a, b, b c\}$ we have $\left|K^{2}\right|=8$ which is a contradiction. Thus $|P|=2$ and $G \cong S_{3}$.

\section{Groups With SMall CUBing on 2-SEts}

In this section we consider finite groups $G$ with the small cubing property on 2 sets, that is $\left|K^{3}\right|<8$ for all 2-element subsets $K$ of $G$. The main result we prove is the following technical theorem.

THEOREM 5.1. Suppose that $G$ is a finite group such that $\left|K^{3}\right|<8$ for all 2-element subsets $K$ of $G$. Then the following hold.

(a) The group $G$ has a normal abelian Hall $\{2,3\}$ '-subgroup $H$.

(b) A Sylow 3-subgroup $P$ of $G$ centralises $H$. Further either $P$ is abelian, or $H=1, P$ is a nonabelian group of exponent 3 and $P$ contains its centraliser in $G$ that is $C_{G}(P)=Z(P)$.

(c) If $Q$ is a Sylow 2-subgroup of $G$ then $\left\langle a^{2} \mid a \in Q\right\rangle$ centralises $H$.

An immediate corollary to this result is the complete classification of odd order groups with the small cubing property on 2-sets stated as Theorem 4 in the introduction.

Unfortunately we do not have sufficient information about the Hall $(2,3)$ subgroups of $G$ to get a classification of the even order examples. This is an open problem. However we are able to obtain the weaker classification, Theorem 5, of finite groups $G$ such that $\left|K^{3}\right|<6$ for all 2-element subsets $K$ of $G$.

First we prove Theorem 5.1 in a sequence of lemmas.

Lemma 5.2. Let $G$ be a finite group, which is not a 2-group, with the small cubing property on 2-sets. Let $p$ be an odd prime dividing $|G|$ and let $P$ be a Sylow $p$-subgroup of $G$. Then either $P$ is abelian, or $p=3, P$ has exponent 3 , there is a 2-element subset $K$ of $P$ with $\left|K^{3}\right|=7$, and the centraliser $C_{G}(P)$ of $P$ is the centre $Z(P)$ of $P$.

Proof: Suppose that $P$ is not abelian and let $b \in P-Z(P)$. Then $b$ lies in a maximal normal subgroup $N$ of $P$, and, as $P$ is generated by $P-N$, there is an element $a \in P-N$ such that $a b \neq b a$. Let $K=\{a, b\}$. Then each of $K^{3} \cap a N=\left\{a b^{2}, b a b, b^{2} a\right\}$ and $K^{3} \cap a^{2} N=\left\{a^{2} b, a b a, b a^{2}\right\}$ has size 3 . Since $\left|K^{3}\right|<8$ it follows that $a^{3}=b^{3}$ whence $p=3$ (for $a b \neq b a$ ) and $\left|K^{3}\right|=7$. Similarly by considering $K=\left\{a, b^{2}\right\}$ we obtain $a^{3}=b^{6}$ whence $a^{3}=b^{3}=1$. Thus all elements in $P-Z(P)$ have order 3 . If now $c \in Z(P)$ then $b c \notin Z(P)$ so $1=(b c)^{3}=b^{3} c^{3}=c^{3}$. Hence $P$ has exponent 3 .

Finally suppose that $c$ is a nontrivial element in $C_{G}(P)-P$ and consider $K=$ $\{a, b c\} \subset P \times\langle c\rangle$. The sets $K^{3} \cap P c^{2}$ and $K^{3} \cap\left(P c \cup P c^{3}\right)$ have sizes 3 and 4 
respectively and it follows that $\left|K^{3}\right|=8$ which is a contradiction. Hence $C_{G}(P)=$ $Z(P)$.

LemMa 5.3. Let $G$ be an odd order group with the small cubing property on 2-sets. Then (a) and (b) of Theorem 5.1 are true.

Proof: Suppose that Theorem 5.1 is false for some odd order group and let $G$ be such a group with minimal order. By Lemma 5.2 it follows that $G$ is not nilpotent. Also, by minimality and Lemma 5.2, each proper subgroup of $G$ is either abelian or a nonabelian group of exponent 3 . Suppose first that a Sylow 3-subgroup $P$ of $G$ is nonabelian. Now $G$ is soluble and $G \neq P$. If $G / G^{\prime}$ is a 3-group, then by minimality $G^{\prime}$ has a normal abelian Hall $\{2,3\}^{\prime}$-subgroup $H$ and $H$ is a Hall subgroup of $G$. By Lemma 5.2, $P$ does not centralise $H$ and so there are noncommuting elements $a \in P$ and $b \in H$. If $K=\{a, b\}$ then $\left|K^{3}\right|=8$ which is a contradiction. Thus $\left|G / G^{\prime}\right|$ is divisible by some prime $p$ greater than 3 . Then $G$ has a maximal normal subgroup $M$ of index $p$. Since $M$ contains $P$ it follows from minimality that $M=P$. Let $c \in G$ have order $p$, and let $K=\{a, b\} \subseteq P$ with $\left|K^{3}\right|=7$ as in the proof of Lemma 5.2. If $c$ centralised $P$ then $\left|\{a, b c\}^{3}\right|=8$ which is a contradiction. Hence $c$ does not centralise $P$, so let $b \in P$ be such that $c b \neq b c$. Then if $K=\{c, b\}$ we have $\left|K^{3}\right|=8$ which is a contradiction. Thus $P$ is abelian and each proper subgroup of $G$ is abelian. Then by [11] or [18,9.1.9], $|G|=p^{u} q^{v}$ for distinct primes $p$ and $q, G$ has a normal abelian Sylow $q$-subgroup $Q$ and a cyclic Sylow $p$-subgroup $\langle a\rangle$. Since $G$ is nonabelian there is an element $b \in Q$ such that $a b \neq b a$. Then if $K=\{a, b\}$ we have $\left|K^{3}\right|=8$ which is a contradiction. This completes the proof of the lemma.

Lemma 5.4. Let $G$ be an even order group with the small cubing property on 2-sets. Then Theorem 5.1 is true for $G$.

Proof: Suppose that Theorem 5.1 is false for some even order group and let $G$ be such a group with minimal order. Suppose that $G$ is not simple and let $N$ be a maximal normal subgroup of $G$. By the minimality of $G$ and by Lemma 5.3, $N$ has a normal Hall $\{2,3\}^{\prime}$-subgroup $H_{1}$. Since $H_{1}$ is a characteristic subgroup of $N$ it follows that $H_{1}$ is normal in $G$. Suppose that $H_{1}$ is nontrivial. Then, by minimality, $G / H_{1}$ has a normal Hall $\{2,3\}$ '-subgroup $H / H_{1}$ where $H \supseteq H_{1}$. It follows that $H$ is a normal Hall $\{2,3\}^{\prime}$-subgroup of $G$, and $H \neq G$ since $|G|$ is even. Then $G / H$ is a $\{2,3\}$-group and, in particular, $G$ is soluble. Let $T$ be a Hall 2 '-subgroup of $G$. Then $T$ contains $H$ and, since $H \supseteq H_{1} \neq\{1\}$ it follows from Lemma 5.3 that $T$ is abelian. Thus $H$ is abelian, a Sylow 3-subgroup $P$ of $G$ is abelian, and $P$ centralises $H$. Let $Q$ be a Sylow 2-subgroup of $G$. If $a^{2}$ centralised $H$ for each $a \in Q$ then Theorem 5.1 would be true for $G$. Thus there are elements $a \in Q$ and $b \in H$ such that $a^{2} b \neq b a^{2}$. But then $\left|\{a, b\}^{3}\right|=8$. Hence $H_{1}=\{1\}$, that is $N$ is a $\{2,3\}$-group. Now by minimality 
and Lemma 5.3 the quotient group $G / N$ satisfies the conclusions of Theorem 5.1. In particular $G / N$ is soluble, and since $G / N$ is simple we have $G / N \simeq Z_{p}$ for some prime p. By minimality a Sylow 3-subgroup of $G$ is abelian or nonabelian of exponent 3 . If $p \leqslant 3$ then Theorem 5.1 is true for $G$ and hence $p \geqslant 5$. Thus $|G|=2^{u} 3^{v} p$ and $N$ is the unique maximal normal subgroup of $G$. Let $M$ be a minimal normal subgroup of $G$. Then by minimality it follows that $G / M$ has a normal Hall $\{2,3\}^{\prime}$-subgroup $L / M$ of order $p$. If $L \neq G$, then, again by minimality, $L$ has a normal subgroup $L_{1}$ of order $p$. Further $L_{1}$ is a normal $\{2,3\}^{\prime}$-subgroup of $G$ and (considering the subgroup $L_{1} P$ ) a Sylow 3-subgroup $P$ is abelian and centralises $L_{1}$. Also for each 2-element $a, a^{2}$ centralises $L_{1}$ (consider $K=\{a, b\}$ for $b \in L_{1}$ ). Hence Theorem 5.1 is true for $G$ which is a contradiction. Thus $L=G$, and it follows that $N=M$ is a minimal normal subgroup of $G$ and hence is an elementary abelian 2-group. Now $G$ acts irreducibly on $N$ and so a subgroup $\langle a\rangle$ of order $p$ is self-normalising in $G$. Thus each element of $G-N$ has order $p$. Let $b \in a^{2} N$. If $K=\{a, b\}$ then $\left|K^{3}\right|=8$ (for $K^{3} \cap a^{4} N=\left\{b a^{2}, a^{2} b, a b a\right\}, K^{3} \cap a^{5} N=\left\{b a b, b^{2} a, a b^{2}\right\}, K^{3} \cap\langle a\rangle=\left\{a^{3}\right\}$, and neither $a$ nor $a^{2}$ commutes with $b$ ).

We conclude that $G$ is a simple group, and as Theorem 5.1 is false for $G, G$ is a nonabelian simple group. By Burnside's Theorem $[12,4.3 .3],|G|$ is divisible by at least three distinct primes, $p, q, r$, say, with $p>q>r$. Then $p>q \geqslant 3$. As $G$ is simple it is generated by its elements of order $p$, and, as $G$ has trivial centre, no non-identity element of $G$ is centralised by all the elements of order $p$. In particular, for $a \in G$ of order $q$, there is an element $b \in G$ of order $p$ such that $a b \neq b a$. Let $K=\{a, b\}$. By our assumption, $\left|K^{3}\right|<8$. Thus $x_{1} x_{2} x_{3}=y_{1} y_{2} y_{3}$, where each $x_{i}$ and $y_{i}$ is $a$ or $b$ and the triples $\left(x_{1}, x_{2}, x_{3}\right)$ and $\left(y_{1}, y_{2}, y_{3}\right)$ are distinct. Since $a b \neq b a$, and since $a$ and $b$ have odd order, we must have $x_{1} \neq y_{1}$. Similarly $x_{3} \neq y_{3}$. We may assume that $x_{1}=a$ and $y_{1}=b$. Suppose first that $x_{3}=a$. Then $y_{3}=b$. If $x_{2}=a$ and $y_{2}=b$, then $\langle b\rangle=\left\langle b^{3}\right\rangle=\left\langle a^{3}\right\rangle$, which is not the case. Thus if $x_{2}=a$ then also $y_{2}=a$ and $a^{3}=b a b$. In this case $a^{4}=(a b)^{2}$, and so $\langle a\rangle=\left\langle a^{4}\right\rangle=\left\langle(a b)^{2}\right\rangle \leqslant\langle a b\rangle$; it follows that $\langle a, b\rangle=\langle a b\rangle$ which contradicts the fact that $a b \neq b a$. Therefore $x_{2}=b$. If also $y_{2}=b$ then $b^{3}=a b a$ and we get a contradiction as before. So $y_{2}=a$ and $a b a=b a b$. Then $(b a)^{-1} a(b a)=(b a)^{-1} b a b=b$, so that $a$ and $b$ are conjugate. This is impossible since $a$ and $b$ have different orders.

Thus $x_{3}=b$ and $y_{3}=a$. Suppose that $x_{2}=a$. Then $a^{2} b=b y_{2} a$. Since $a b \neq b a$ and $a$ has odd order, $a^{2} b \neq b a^{2}$, and therefore $y_{2}=b$ and $a^{2} b=b^{2} a$. It follows that $b^{-1} a^{2} b=b^{-1} b^{2} a=b a$, and $a^{-1} b^{2} a=a b$. Thus $b^{-2} a^{2} b^{2}=b^{-1} b a b=a b=a^{-1} b^{2} a$, which means that $a^{2}$ and $b^{2}$ are conjugate. This is impossible as $a^{2}$ and $b^{2}$ have different orders. Hence $x_{2}=b$ and $a b^{2}=b y_{2} a$. Again, as $a b^{2} \neq b^{2} a$, we must have $y_{2}=a$. Then $a b^{2}=b a^{2}$, and hence $\left(b^{-1}\right)^{2} a^{-1}=\left(a^{-1}\right)^{2} b^{-1} ;$ this leads to a 
contradiction as above. This completes the proof of Lemma 5.4.

Lemmas 5.3 and 5.4 complete the proof of Theorem 5.1. Now we prove Theorem 5.

Proof of Theorem 5: First we show that abelian groups and groups satisfying (a) and (b) have the property that $\left|K^{3}\right|<6$ for all 2-element subsets $K$. This is certainly true for abelian groups. Next let $G=\langle a, H\rangle$ where $H$ is abelian of exponent 4 and of index 2 in $G, a^{2}=1$, and $a x a=x^{-1}$ for all $x$ in $H$. Let $K$ be a 2-element subset of $G$. If $K \subseteq H$ then $\left|K^{3}\right| \leqslant 4$ so assume that $K$ contains an element $c$ of $G-H$. Now $c=a y$ for some $y$ in $H$. We have $c^{2}=y^{a} y=1$ and if $x \in H$ then $x^{c}=\left(x^{a}\right)^{y}=x^{-1}$ so we may assume that $c=a \in K$. If $K=\{a, x\}$ with $x \in H$ then $K^{3}=\left\{a=a^{3}=x a x, x=x^{3}=a x a=a^{2} x=x a^{2}, a x^{2}, a x^{2}=x^{2} a\right\}$ has order at most 3. Thus we may assume that $K=\{a, a x\}$ for some $x \in H$. Then $K^{3}=\left\{a=a^{3}=\right.$ $\left.(a x)^{2} a=a(a x)^{2}, a x=a^{2}(a x)=(a x) a^{2}=(a x)^{3}, a x^{2}=(a x) a(a x), a x^{3}=a(a x) a\right\}$ has order at most 4 . Finally let $G$ be a 2 -group such that $\Phi(G)=\langle x\rangle$ has order 2 . Then $x$ is in the centre of $G$. Let $K$ be a 2-element subset of $G$. If $x \in K$ then $\left|K^{3}\right|<6$ so assume that $K=\{a, b\} \subseteq G-\langle x\rangle$. Then $a^{2}$ and $b^{2}$ lie in $\Phi(G)=\{1, x\} \subseteq Z(G)$ and each element of $K^{3}$ is equal to one of $a, a x, b, b x$. Thus each of the groups in the conclusion of Theorem 5 has the required cubing property.

Now suppose that $G$ is a nonabelian group with the property that $\left|K^{3}\right|<6$ for all 2-element subsets $K$. Then $G$ satisfies the conclusions of Theorem 5.1. By Lemma 5.2 a Sylow 3-subgroup $P$ of $G$ is abelian and so a Hall 2 '-subgroup $H P$ of $G$ is abelian. Since $G$ is not abelian $|G|$ is even. Let $Q$ be a Sylow 2 -subgroup of $G$. If there are elements $a \in Q$ and $b \in H$ such that $a b \neq b a$, then, with $K=\{a, b\}$, each of the sets $K^{3} \cap\left(H \cup a^{2} H\right)$ and $K^{3} \cap\left(a H \cup a^{3} H\right)$ has size at least 3, contradicting the fact that $\left|K^{3}\right|<6$. Thus $Q$ centralises $H$, whence $H$ lies in the centre $Z(G)$ of $G$. Suppose that $G$ has a normal subgroup $M$ of index 3 . Then, by minimality, either $M$ is abelian or $M$ is a nonabelian 2-group. We claim that $G$ has a section isomorphic to $A_{4}$. Let $G=\langle M, c\rangle$ with $c \in P$. As $G$ is not abelian, and as $c$ centralises $P H$, in either case $c$ normalises but does not centralise $Q$. It follows that some section of $\langle Q, c\rangle$ is isomorphic to $A_{4}$. However $\left\{\left(\begin{array}{lll}1 & 2 & 3\end{array}\right),\left(\begin{array}{ll}1 & 2\end{array}\right)\left(\begin{array}{ll}3 & 4\end{array}\right)\right\}^{3}$ has order 7 . Thus $G$ has no normal subgroup of index 3 , and therefore a maximal normal subgroup $M$ of $G$, with $B \leqslant M$, has index 2 in $G$. Suppose now that $P H \neq 1$. Then, by minimality, $M$ is abelian, whence $M=P H \times(M \cap Q)$. Let $G=\langle M, c\rangle$ with $c \in Q$. If $c a \neq a c$ for some $a \in P H$, then, with $K=\{a, c\}$, each of the sets $K^{3} \cap\left(P H \cup a^{2} P H\right)$ and $K^{3} \cap\left(a P H \cup a^{3} P H\right)$ has size at least 3 , contradicting $\left|K^{3}\right|<6$. Thus $c$ centralises $P H$ and we have $G=P H \times Q$, whence $Q$ is nonabelian and $c a \neq a c$ for some $a \in Q$. Then, if $b \in(P H) \backslash\{1\}$ and $K=\{a b, c\}$, we have $\left|K^{3}\right| \geqslant 6$ (noting that the sets $\left\{c^{3}, a^{3} b^{3}\right\},\left\{(a b)^{2} c, a b c a b\right\}$, and $\left\{a b c^{2}, c a b c\right\}$ are each of size 2 and are disjoint, as they lie in disjoint unions of cosets of $Q$ ). Thus $P H=1$ and $G$ is a nonabelian 2-group. 
If $\left|K^{2}\right|<4$ for all 2-element subsets of $G$ then Theorem 5(b) is true by [9] so we may assume that for some $K=\{a, b\}$ we have $\left|K^{2}\right|=4$. For such 2-element subsets $K$ we consider Table 1 below,

\begin{tabular}{l|llll} 
& $a^{2}$ & $a b$ & $b a$ & $b^{2}$ \\
\hline$a$ & $a^{3}$ & $a^{2} b$ & $a b a$ & $a b^{2}$ \\
$b$ & $b a^{2}$ & $b a b$ & $b^{2} a$ & $b^{3}$
\end{tabular}

Table 1

called a third stage multiplication table in [4]. We write $A=a^{3}, B=a^{2} b, C=a b a$, $D=a b^{2}$. Then according to the analysis in [4], since $\left|K^{2}\right|=4$ and $\left|K^{3}\right|<6$, the second row $\left(b a^{2}, b a b, b^{2} a, b^{3}\right)$ of Table 1 is one of $(B, A, D, C),(D, A, E, C)$, $(D, C, B, A)$ and $(E, A, B, C)$ where $E \in G$ is distinct from $A, B, C, D$. Let us temporarily call these types $1,2,3$ and 4 respectively. We will show that only type 1 is possible.

Suppose first that $K$ is of type 2 , that is $a^{3}=b a b, a b a=b^{3}$, and $a b^{2}=b a^{2}$. It follows that, for $L=\left\{a^{2}, b\right\},\left|L^{2}\right|=4$. If $L$ is of type 1,2 or 4 , then $a^{6}=b a^{2} b$ which in turn is equal to $a b^{3}$, whence $a^{5}=b^{3}=a b a$ so that $b=a^{3}$ and hence $a b=b a$, which is a contradiction. Thus $L$ is of type 3 , so $a^{6}=b^{3}$ which equals $a b a$, whence $a^{4}=b$ and $a b=b a$, again a contradiction.

Next suppose that $K$ is of type 3 , that is $a b^{2}=b a^{2}, a b a=b a b, a^{2} b=b^{2} a$, and $a^{3}=b^{3}$. Again it follows that $\left|L^{2}\right|=4$ for $L=\left\{a^{2}, b\right\}$. If $L$ is of type 1,2 or 4 then $a^{6}=b a^{2} b$ which equals $a b^{3}$, whence $a^{5}=b^{3}=a^{3}$ and so $a^{2}=1$ which contradicts the fact that $\left|L^{2}\right|=4$. Thus $L$ is of type 3 so $a^{2} b^{2}=b a^{4}$ which equals $a b^{2} a^{2}=a b a b^{2}$ whence $b=1$ which is a contradiction.

Finally suppose that $K$ is of type 4 , that is $a^{3}=b a b, a^{2} b=b^{2} a$, and $a b a=$ $b^{3}$. Again we find that $\left|L^{2}\right|=4$ for $L=\left\{a^{2}, b\right\}$ and arguing as above leads to a contradiction.

Thus any $K=\{a, b\}$ such that $\left|K^{2}\right|=4$ is of type 1 , that is $a^{3}=b a b, a^{2} b=b a^{2}$, $a b^{2}=b^{2} a, a b a=b^{3}$. Since for such a set $K=\{a, b\}$ we have $a b \neq b a$, certainly $a^{2} b \neq$ $a b a$. If $a^{2} \neq(a b)^{2}$ then $L=\{a, a b\}$ is also of type 1 and so $a^{3}=(a b) a(a b)=a^{3} b^{2}$, that is $b^{2}=1$. On the other hand if $a^{2}=(a b)^{2}$ then $a^{2}=a(b a b)=a^{4}$, that is $a^{2}=1$. Thus, for each $K=\{a, b\}$ such that $\left|K^{2}\right|=4$, either $a^{2}=1$ or $b^{2}=1$.

We claim that $G$ has exponent 4 . Let $K=\{a, b\}$ have $\left|K^{2}\right|=4$ and suppose that $b^{2}=1$. Then $a^{6}=(b a b)^{2}=b a^{2} b=a^{2}$ so that $a^{4}=1$, and as $a^{2} \neq b^{2}=1$, $a$ has order 4 .

Now suppose that there is an $x \in G$ with $x^{4} \neq 1$. Then $\left|\{a, x\}^{2}\right|<4$ and as $x^{2}$ (of order at least 4 ) is not equal to $a^{2}$ we must have $a x=x a$. Similarly $b x=x b$. But then $\left|\{a, b x\}^{2}\right|=4$ while $a^{2} \neq 1$ and $(b x)^{2}=x^{2} \neq 1$, which is a contradiction. Thus $G$ has exponent 4 . 
Suppose that, for each pair $a, b$ of non-commuting elements of $G$ either $a^{2}=1$ or $b^{2}=1$. Then $A=\left\langle x \mid x^{2} \neq 1\right\rangle$ is an abelian subgroup of $G$. Let $a \in G-A$. Then $a^{2}=1$, and if $x \in A$ then $a x \in G-A$ and so $(a x)^{2}=1$, that is $x^{a}=x^{-1}$. Thus each element of $G-A$ inverts $A$. Further $G / A$ is elementary abelian. If $|G / A| \geqslant 4$ then there are elements $a, b, a b$ in $G-A$ lying in distinct cosets of $A$ and it is not possible for each of $a, b$ and $a b$ to invert $A$. Thus $|G: A|=2$. Finally, as $G$ has exponent 4, $A$ has exponent 4 and so $G$ satisfies (b)(i) of the theorem.

Thus we may suppose that there is a pair $a, b$ of non-commuting elements such that $a^{2}=b^{2} \neq 1$. We claim that $\Phi(G)=\left\langle x^{2} \mid x \in G\right\rangle$ has order 2. Suppose that there is an $x \in G$ such that $1 \neq x^{2} \neq a^{2}$. Then $\left|\{a, x\}^{2}\right|<4$ and so $a x=x a$. Similarly $b x=x b$. Also $(b x)^{2}=b^{2} x^{2}=a^{2} x^{2} \neq a^{2}$ and $(b x) a=b a x \neq a b x$ and so $\left|\{a, b x\}^{2}\right|=4$ while $a^{2} \neq 1$ and $(b x)^{2}=a^{2} x^{2} \neq 1$. This is a contradiction and so $\Phi(G)=\left\langle a^{2}\right\rangle$ has order 2 and (b)(ii) of the theorem is true. This completes the proof of Theorem 5.

REMARK. Nekrasov [16] proved that a finite nonabelian group with the property that, for each $K=\{a, b\}$ with $\left|K^{2}\right|=4$, the equalities $a^{3}=b a b, a^{2} b=b a^{2}, a b^{2}=b^{2} a$ and $a b a=b^{3}$ hold, satisfies part (b) of Theorem 5; the last part of our proof of Theorem 5 is basically due to him.

\section{REFERENCES}

[1] Ya. G. Berkovich, 'On p-groups of finite order', Siberian Math. J. 9 (1968), 1284-1306. (in Russian; English translation).

[2] Ya. G. Berkovich, 'Generalization of theorems of P. Hall and Blackburn and an application to non-regular p-groups', Izv. Akad. Nauk SSSR Ser. Mat. 35 (1971), 800-831. (in Russian; English translation).

[3] Ya. G. Berkovich and G.A. Freiman, 'Group structure and cardinality of powers of its small subsets', (unpublished manuscript, 1981).

[4] L.V. Brailovsky and G.A. Freiman, 'On two-element subsets in groups', Ann. New York Acad. Sci. 373 (1981), 183-190.

[5] L.V. Brailovsky and G.A. Freiman, 'On a product of finite subsets in a torsion-free group', J. Algebra 130 (1990), 462-476.

[6] L.V. Brailovsky and G.A. Freiman, 'Groups with small cardinality of their two-element subsets', Ann. New York Acad. Sci 410 (1983), 75-82.

[7] G.A. Freiman, Foundations of a structural theory of set addition, (in Russian) (Kazan, 1966). Translations of Mathematical Monographs 37, (English translation) (Amer. Math. Soc., Providence, R.I., 1973).

[8] G.A. Freiman, 'Groups and the inverse problems of the additive set theory', in Number-theoretic investigations on the Markov spectrum and the structure theory of set addition, (in Russian), pp. 175-183 (Kalinin University, Moscow, 1973).

[9] G.A. Freiman, 'On two- and three-element subsets of groups', Aequationes Math. 22 
(1981), 140-152.

[10] G.A. Freiman and B.M. Schein, 'Group and semigroup theoretic considerations inspired by inverse problems of the additive number theory', in Lecture Notes in Math. 1320, pp. 121-140 (Springer-Verlag, Berlin-Heidelberg-New York, 1988).

[11] J.A. Golfand, 'On groups all of whose subgroups are special', Dokl. Akad. Nauk SSSR) 60, No. 8 (1948), 1313-1315.

[12] D. Gorenstein, Finite groups (Harper and Row, New York, 1968).

[13] B. Huppert, Endliche gruppen I (Springer-Verlag, Berlin, Heidelberg, New York, 1967).

[14] J.H.B. Kemperman, 'On complexes in a semigroup', Indag. Math. 18 (1956), 247-254.

[15] K.G. Nekrasov and Ya.G. Berkovich, 'A necessary and sufficient condition for the cyclicity of the Frattini subgroup of a finite p-group, Voprosyteorii Grup, Gomologicheskoi algebry, Yaroslarl', pp. 55-57, (1985).

[16] K.G. Nekrasov, private communication, 1987.

[17] P.M. Neumann, private communication, 1989.

[18] D.J.S. Robinson, $A$ course in the theory of groups (Springer-Verlag, Berlin, Heidelberg, New York, 1982).

Department of Mathematics

Bar-Ilan University 52900 Ramat-Gan

Israel

Department of Mathematics

University of Western Australia

Nedlands WA 6009

Australia
School of Mathematical Sciences

Raymond and Beverley Sackler

Faculty of Exact Sciences

Tel-Aviv University

Ramat - Aviv 69978

Israel 